\title{
Using the attitude response of aerostable spacecraft to measure thermospheric wind
}

DOI:

10.1007/s12567-017-0153-9

\section{Document Version}

Accepted author manuscript

Link to publication record in Manchester Research Explorer

\section{Citation for published version (APA):}

Virgili Llop, J., Roberts, P., \& Hao, Z. (2017). Using the attitude response of aerostable spacecraft to measure thermospheric wind. CEAS Space Journal, 1-13. https://doi.org/10.1007/s12567-017-0153-9

\section{Published in:}

CEAS Space Journal

\section{Citing this paper}

Please note that where the full-text provided on Manchester Research Explorer is the Author Accepted Manuscript or Proof version this may differ from the final Published version. If citing, it is advised that you check and use the publisher's definitive version.

\section{General rights}

Copyright and moral rights for the publications made accessible in the Research Explorer are retained by the authors and/or other copyright owners and it is a condition of accessing publications that users recognise and abide by the legal requirements associated with these rights.

\section{Takedown policy}

If you believe that this document breaches copyright please refer to the University of Manchester's Takedown Procedures [http://man.ac.uk/04Y6Bo] or contact uml.scholarlycommunications@manchester.ac.uk providing relevant details, so we can investigate your claim.

\section{OPEN ACCESS}




\title{
Using the attitude response of aerostable spacecraft to measure thermospheric wind
}

\author{
Josep Virgili-Llop • Peter C.E. Roberts • Zhou Hao
}

the date of receipt and acceptance should be inserted later

\begin{abstract}
In-situ measurements of the thermospheric wind can be obtained by observing the attitude response of an aerostable spacecraft. In the proposed method, the aerostable spacecraft is left uncontrolled, freely reacting to the aerodynamic torques, and oscillating around its equilibrium attitude. The wind's magnitude and direction is determined by combining the attitude observations with estimates of the other perturbing torques, atmospheric density, and spacecraft's aerodynamic properties. The spatial resolution of the measurements is proportional to the natural frequency of the attitude's oscillation. Spacecraft with high aerodynamic stiffness to inertia ratios operating at low altitudes exhibit higher natural frequencies, making them particularly suited for this method. A one degree-offreedom case is used to present and illustrate the proposed method as well as to analyze its performance.
\end{abstract}

Keywords thermospheric wind · estimation - spacecraft aerodynamics

\section{Introduction}

Thermospheric wind plays an important role in the electrodynamics of the upper atmosphere (via the ion and neutral particle interaction). Despite its importance, the knowledge of the thermospheric wind is still rather

Josep Virgili-Llop

Naval Postgraduate School

1 University Circle, Monterey, CA, 93940, USA

Tel.: +1 831-233-2879

E-mail: jvirgili@nps.edu

Peter C.E. Roberts and Zhou Hao

University of Manchester

Sackville Street, Manchester, M13 9PL, UK limited. This incomplete knowledge is caused, in part, by an insufficient number of wind measurements [1,2].

In-situ and remote sensing wind measurement methods do exist. In-situ methods include chemical tracers delivered by sounding rockets as well as high-precision spacecraft-mounted accelerometers. For remote sensing, space- or ground-based, interferometers and incoherent scatter radar techniques are used. A brief survey of these methods is provided in Section 2 .

The proposed measurement approach differs from existing methods, as it relies on the perturbing effect that the wind has on a spacecraft's attitude. The challenge of the proposed method lies in discerning the effect that the wind has on the spacecraft's attitude evolution. Once its effects are isolated, the wind's magnitude and direction can be determined.

For this method, spacecraft sensitive to aerodynamic torques are preferred, as the "signal" induced by the aerodynamic torques is larger and easier to measure. To reduce the "noise", the rest of the torques, including control torques, need to be minimized. This combination prompts the use of aerostable spacecraft, which can be made very sensitive to aerodynamic torques and left uncontrolled, oscillating around their equilibrium attitude.

The perturbing effects of the aerodynamic torques are not only due to wind. The spacecraft's aerodynamic properties, the atmospheric density, and the spacecraft's inertial velocity also play a significant role. In addition, the attitude evolution is not only driven by aerodynamic torques, other residual perturbing effects (e.g., solar radiation pressure, structural flexibility, etc.) account for some of the observed rotational motion. Estimates of these quantities are required in order to discern the wind's effect. 
The method that more closely resembles the proposed one is the accelerometer-base approach. That method uses the accelerations induced by the aerodynamic forces to determine the wind's magnitude and direction. The proposed approach uses the angular acceleration instead. As "low-cost" and miniaturized Commercial-off-the-Shelf (COTS) high-precision attitude sensors (i.e., star trackers) are readily available, while high-precision accelerometers are custom buildwith their associated cost penalty - we can venture to suggest that the proposed method may offer several implementation advantages.

In order to help understand the principle of operation and performance of the different currently available wind measurement methods, this paper opens with a brief survey of these wind measurement methods. An overview of the proposed method follows. For simplicity, the analysis is strictly restricted to a one-dimensional case. Two different approaches to retrieve the wind from attitude observations are presented, an iterative and a frequency approach. Finally a case study is presented where, for an ideal case without uncertainties, the accuracy of the two different approaches is derived.

\section{Brief Survey of Thermospheric Wind Measurement Methods}

Studying the atmosphere by observing the effects that the aerodynamic forces have on the spacecraft dynamics has been done since Sputnik 1 [3. By observing the change in the orbit's inclination, it can be determined that the atmosphere co-rotates and that average WestEast winds are in the order of $100 \mathrm{~m} / \mathrm{s}$. This was first noticed by observing how Sputnik 2 orbital inclination decreased from its initial $65.33^{\circ}$ to $65.19^{\circ}$ at the end of its life 4. Many similar observations followed afterwards. Although the results of this type of analysis are too geographically and time averaged to be useful to advance atmospheric science, they are nonetheless important within the orbital dynamics field [5].

Sounding rockets have traditionally been used to take in-situ measurements of the upper atmosphere wind 6]. These rockets release a chemical tracer (usually sodium, lithium, or trimethyl aluminum) as they ascend (or descend) through the atmospheric layers of interest. The chemical tracers produce visible light allowing to track their apparent motion and thus infer the wind velocities. Sodium and lithium tracers produce resonant emissions that only occur when the vapor is illuminated. Therefore, measurements can only be made when the trails are illuminated by the Sun but the observers on the ground are in darkness (only viable at dusk or dawn). Trimethyl aluminum reacts with the atmospheric oxygen to produce chemiluminescence, so observations at night are possible. Optical tracking of the trails provides wind profiles with good height resolution, generally in $0.5 \mathrm{~km}$ increments or better, and relatively small uncertainties, generally below $5 \mathrm{~m} / \mathrm{s} 2$. As of 2002, approximately over 500 chemical tracer measurements have been made since 1958, with the vast majority at the 80 to $180 \mathrm{~km}$ altitude range [6, 2].

In-situ measurements have also been performed by spacecraft measuring the accelerations caused by aerodynamic forces. Although these acceleration-based methods have only been able to retrieve the cross-track component $[7,8,9$, they have been successfully used with the data provided by the CHAMP, GRACE, and GOCE accelerometers (and even using the Mars Global Surveyor and Odyssey data on the Martian atmosphere [10]).

Accelerometer-based measurements are usually limited to cross-track wind as the acceleration measurement is also used to estimate the atmospheric density. From the in-track acceleration data alone it is impossible to distinguish the acceleration caused by the in-track wind from the acceleration caused by density variations (as both affect the dynamic pressure). As the magnitude of the in-track wind is small when compared to the spacecraft's inertial velocity, the in-track wind can be safely neglected when determining the atmospheric density [9]. If a different source of density measurements could be used (e.g., from a mass spectrometer) then the in-track wind effects could be discriminated and measured [11].

Of particular relevance to the accelerometer-based method is the work that has been recently conducted using CHAMP's accelerometer data [1, 12, 13, 14, 15. Wind measurements derived from CHAMP's data have a wind magnitude resolution of about $\pm 10 \mathrm{~m} / \mathrm{s}$ and a spatial resolution of $76 \mathrm{~km} 13$. Work from the GOCE's datasets has also started to appear in the literature [16. 17. and the ongoing SWARM mission is also expected to yield thermospheric wind measurements [18.

Finally, spectrometers onboard spacecraft have also been used to in-situ measure the thermospheric wind. Mass spectrometers can directly measure the angle of arrival of a beam of particles entering the spectrometer's slotted orifice [19].

Remote sensing techniques using ground- or spacebased instruments have also been extensively employed. These use either interferometer or incoherent scatter radar techniques. On the interferometer front, FabryProt Interferometers (FPI) are the most common and have been widely employed to measure the thermospheric wind both from space and from the ground. An FPI observes the Doppler shift of the airglow emitted by 
different chemical species (mainly atomic oxygen) [20]. As the airglow emissions occur at specific wavelengths (e.g., at $6300 \AA$ for atomic oxygen $\mathrm{O}\left({ }^{1} \mathrm{D}\right)$ ), accurate Doppler measurements can be taken and the velocity of these gas particles inferred. As it is impossible to obtain the three components of the wind simultaneously, from a single measurement, ground-based instruments generally assume that the vertical component of the wind is negligible. By taking measurements at the four cardinal points (i.e., $\mathrm{N}-\mathrm{E}-\mathrm{S}-\mathrm{W}$ ), the overhead zonal and meridional wind components can be obtained (as these are the difference between the North-South and East-West measurements) [21]. If the instrument is particularly well calibrated, the vertical component can be obtained by taking a direct zenith measurement [21]. Multiple instruments observing the same location have been often used to obtain instantaneous zonal and meridional wind measurements 22]. Although FPIs are certainly the most common interferometers, Michelson interferometers have also been used [23. Ground based FPIs have an uncertainty on their measurements of roughly $\sim 2-30$ $\mathrm{m} / \mathrm{s}$ [24,25] during nighttime and $\sim 70 \mathrm{~m} / \mathrm{s}$ during daytime [26]. In 2006 there were less than 15 ground-based FPIs over the entire world [27].

The NASA's Dynamic Explorer-2 (DE-2) launched in 1981 combined the results of its FPI and in-situ Wind and Temperature Spectrometer to obtain full vector wind measurements [28]. With its polar orbit, the DE2 FPI was capable of measuring the meridional winds (North-South) [29]. The Wind and Temperature Spectrometer measured the angle of arrival of the beam of neutral gas particles entering the spectrometer, thus measuring the zonal winds (West-East) 30. Reliable wind measurements could only be made around the orbit's perigee. Due to the highly eccentric orbit only one polar region could be sampled during a given season [12.

Another spacecraft that has used the interferometer technique is the the Upper Atmosphere Research Satellite (UARS) 23]. On board this spacecraft was the Wind Imaging Interferometer (WINDII), a Michelson interferometer measuring Doppler shifts of the green $(557.7 \mathrm{~nm})$ and red line $(630.0 \mathrm{~nm})$ airglow emissions at the Earth's limb. This instrument had the capability of measuring the wind within the $80-300 \mathrm{~km}$ altitude range in a single image with an accuracy of about $1 \mathrm{~km}$ (limited by the stability of the UARS platform).

UARS also carried the High Resolution Doppler Imager (HRDI), a triple-etalon FPI 31,32. Observing the emissions and adsorption lines of molecular oxygen it was capable of measuring the winds in small volumes (4 km in height by $50 \mathrm{~km}$ in width) at about $70-120$ $\mathrm{km}$ altitude range with an accuracy of approximately 5 $\mathrm{m} / \mathrm{s}$.

The TIMED spacecraft had also a limb-scanning FPI named TIMED Doppler Interferometer (TIDI) 33. This instrument could measure daytime and nighttime winds at the $85-105 \mathrm{~km}$ altitude band and had a vertical resolution $2.5 \mathrm{~km}$, with an accuracy approaching $\sim 3 \mathrm{~m} / \mathrm{s}$ under optimum viewing conditions.

Measurements of thermospheric wind using Incoherent Scatter Radar (ISR) have been done for more than 20 years [34]. At the vicinity of Kiruna, in Sweden $\left(70^{\circ} \mathrm{N}, 19^{\circ} \mathrm{E}\right)$, the EISCAT ISR measures the wind by emitting a signal and subsequently measuring the Doppler shift of that return signal backscattered by ionospheric plasma. The EISCAT can measure the wind in a wide range of altitudes (from 180-360 km) and its results agree within $10 \mathrm{~m} / \mathrm{s}$ from those obtained by WINDII 35, 36].

\section{Proposed Method: One-Dimensional Analysis}

The method introduced in this paper is conceptually similar to the accelerometer-based wind measurement method [9]. Instead of relying on the linear acceleration, the proposed method exploits the rotational motion caused by wind-induced aerodynamic torques. As in the accelerometer-based approach, the proposed method can obtain cross-track wind measurements, and if another source of atmospheric density is available, it is also capable of measuring the in-track wind component.

In the proposed method the spacecraft's attitude motion is observed and the torques that shape this motion are determined. By estimating and discounting the other perturbing torques, the aerodynamic torques are isolated. Finally, if the atmospheric density, the spacecraft's inertial velocity, and the spacecraft's aerodynamic properties are known, the wind's magnitude and direction can be determined from the aerodynamic torques.

To better isolate the aerodynamic torques from the other perturbations, it is desirable to have large aerodynamic torques, while minimizing the other perturbing torques -including control torques. Within these preferences, an aerostable spacecraft appears to be a favorable design. Aerostable spacecraft can be very sensitive to aerodynamic forces while not requiring any attitude control, as they naturally oscillate around their equilibrium attitude.

In this analysis, an aerostable spacecraft is defined as a space vehicle that exhibits a restoring aerodynamic torque when it departs from its aerodynamic equilibrium attitude. Due to the high velocity of the relative 


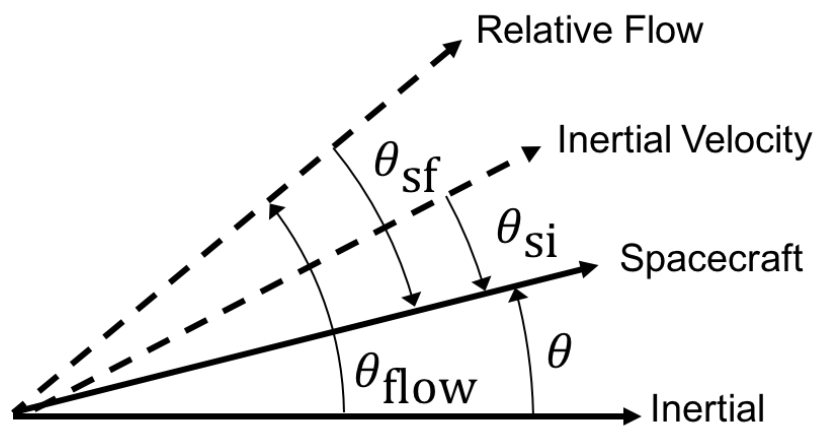

Fig. 1: Angle definitions for a one degree-of-freedom case.

flow, the effect of the spacecraft's angular velocity on the aerodynamic torque is negligible. Once perturbed, an aerostable spacecraft oscillates around its equilibrium attitude. Although the aerodynamic torque can be considered as a conservative torque, the spacecraft's structural flexibility introduces a small damping effect.

In this section, the proposed wind measurement method is derived for a one rotational degree-of-freedom case. With only one degree-of-freedom it is theoretically possible to recover the in-track wind component and one of the cross-track components (either the horizontal or the vertical). This analysis could be extended to a multidimensional case and thus develop the method to recover the remaining cross-track component (see Section 5.

In this one rotational degree-of-freedom scenario, let $\theta_{\text {sf }}$ denote the attitude of the spacecraft with respect to the relative flow and $\theta_{\mathrm{si}}$ its attitude with respect to the inertial velocity. The attitude with respect to an arbitrary inertial frame is simply denoted by $\theta$ and the direction of the relative flow with respect to this inertial frame is denoted by $\theta_{\text {flow }}$, so $\theta-\theta_{\text {sf }}=\theta_{\text {flow }}$. Figure 1 illustrates these angles.

It is assumed that $\theta$ and $\theta_{\text {si }}$ are known (e.g., using star-trackers and a GPS to determine the inertial velocity direction and magnitude). Additionally, the position of the spacecraft is also assumed to be known (e.g., using a GPS receiver) and so the atmosphere co-rotation at that particular point can be easily computed and discounted. The attitude of the spacecraft with respect to the combined inertial velocity and co-rotation $\theta_{\text {sico }}$ can then be derived and it is assumed to be known. Therefore, the difference between the attitude with respect the incident relative flow $\theta_{\text {sf }}$ and the inertial velocity plus co-rotation $\theta_{\text {sico }}$ is only due to the unknown atmospheric wind.

\subsection{Spacecraft dynamics}

In rarefied-gas aerodynamics, the aerodynamic torque, in a one degree-of-freedom case, can be computed as follows 37:

$\tau_{\text {aero }}=\frac{1}{2} \rho\left\|\boldsymbol{v}_{\mathrm{f}}\right\|^{2} A_{\text {ref }} l_{\text {ref }} C_{T}$

with $\rho$ denoting the atmospheric density, $\boldsymbol{v}_{\mathrm{f}}$ the bulk velocity of the gas particles relative to the spacecraft and $A_{r e f}, l_{\text {ref }}$ denoting an arbitrarily defined reference area and length, respectively (usually tied to spacecraft dimensions). The $C_{T}$ term, known as the torque coefficient, is a dimensionless coefficient that combines the spacecraft aerodynamic properties (shape and surface properties), flow properties (composition and temperature), and the orientation of the spacecraft with respect to the flow $\theta_{\text {sf }}$.

Assuming that all the spacecraft and flow parameters remain constant, $C_{T}$ is only a function of the spacecraft attitude with respect to the flow $C_{T}\left(\theta_{\mathrm{sf}}\right)$. Note that the relative flow also includes the wind, making its direction unknown a priori. It is also important to point that the relative flow velocity $\boldsymbol{v}_{\mathrm{f}}$ is composed by the inertial velocity $\boldsymbol{v}_{\mathrm{i}}$, the atmospheric co-rotation velocity $\boldsymbol{v}_{\text {co }}$ and the wind velocity $\boldsymbol{w}$, making $\boldsymbol{v}_{\mathrm{f}}$ also unknown a priori.

$\boldsymbol{v}_{\mathrm{f}}=\boldsymbol{v}_{\mathrm{i}}-\boldsymbol{v}_{\mathrm{co}}-\boldsymbol{w}$

To simplify the analysis and the notation, and without loss of generality, it is assumed that the equilibrium attitude of the aerostable spacecraft is $\theta_{\mathrm{sf}}^{\mathrm{eq}}=0$. With this assumption, the restoring aerodynamic torque can be expressed as

$\tau_{\text {aero }}=-q f\left(\theta_{\mathrm{sf}}\right)$

with $q$ denoting the dynamic pressure

$q=\frac{1}{2} \rho\left\|\boldsymbol{v}_{\mathrm{f}}\right\|^{2}$

The function $f\left(\theta_{\mathrm{sf}}\right)$ can be determined by characterizing the aerodynamic properties of the spacecraft. To simplify the analysis it is assumed that the restoring torque is proportional to the misalignment with respect to the equilibrium attitude $\theta_{\mathrm{sf}}-\theta_{\mathrm{sf}}^{\mathrm{eq}}$. This assumption is generally valid for small misalignments with respect to the equilibrium attitude $\theta_{\mathrm{sf}}^{\mathrm{eq}}$. With these assumptions, the restoring torque can be written, for a onedimensional case as

$\tau_{\text {aero }}=-q k\left(\theta_{\mathrm{sf}}-\theta_{\mathrm{sf}}^{\mathrm{eq}}\right)=-q k \theta_{\mathrm{sf}}$

with $k$ denoting the aerodynamic stiffness, which takes into account the spacecraft's aerodynamic properties 
(the relative flow bulk properties are contained in the dynamic pressure $q$ ). Note how an ideal aerostable spacecraft with a linear restoring torque is analogous to a mass spring system. $q k$ is the spring stiffness equivalent and $\theta_{\text {sf }}$ is the equivalent to the spring's displacement.

If the aerodynamic properties of the spacecraft ( $k$ or $\left.f\left(\theta_{\text {sf }}\right)\right)$, the density $\rho$, the inertial $\boldsymbol{v}_{\text {i }}$ and atmospheric co-rotation velocities $\boldsymbol{v}_{\text {co }}$, as well as the aerodynamic torque $\tau_{\text {aero }}$ are known, the direction of the relative flow $\theta_{\text {sf }}$ and its magnitude $\left\|\boldsymbol{v}_{\mathrm{f}}\right\|$ can be computed using Eq. (5) and Eq. (2). As all the other magnitudes are known, the wind's direction and magnitude can be immediately derived from $\theta_{\text {sf }}$ and $\left\|\boldsymbol{v}_{\mathrm{f}}\right\|$.

By observing the inertial attitude $\theta$ evolution, the angular velocity $\dot{\theta}$, and the angular acceleration $\ddot{\theta}$, the total torque $\tau_{\text {tot }}$ acting on a spacecraft can be computed using Euler's equations of motion. The Euler's equations, simplified for a one degree-of-freedom case, can be simply written as

$\tau_{\text {tot }}=J \ddot{\theta}$

with the known spacecraft inertia along the considered rotational degree-of-freedom denoted by $J$. The total torque $\tau_{\text {tot }}$ does include the aerodynamic torque $\tau_{\text {aero }}$, but also other perturbing torques, namely: the gravity gradient $\tau_{\text {grav }}$, the solar radiation pressure $\tau_{\mathrm{SRP}}$, the residual magnetic dipole $\tau_{\text {mag }}$, the actuators torque $\tau_{\text {actuators }}$ and any torques cause by the spacecraft's structural flexibility $\tau_{\text {flex }}$.

$\tau_{\text {tot }}=\tau_{\text {aero }}+\tau_{\text {grav }}+\tau_{\text {SRP }}+\tau_{\text {mag }}+\tau_{\text {actuators }}+\tau_{\text {flex }}$

These other torques need to be known, or at least estimated, before $\tau_{\text {aero }}$ can be computed (using Eq. 7). A high $\tau_{\text {aero }}$ in combination with low perturbing torqueseffectively rising the "signal-to-noise ratio" - is highly desirable. To achieve a high $\tau_{\text {aero }}$ a high aerodynamic stiffness $k$ is required.

A way to completely eliminate the control torque $\tau_{\text {actuators }}=0$ is to leave the spacecraft uncontrolled. With no active control, an aerostable spacecraft remains in a bounded oscillation around its aerodynamic equilibrium point (as long as the other perturbations are smaller than the aerodynamic torques), with the spacecraft's structural flexibility providing some small level of damping.

The attitude oscillation is so intrinsic to the behavior of uncontrolled aerostable spacecraft that it needs to be taken into account when determining the wind components. Two different approaches that leverage this oscillatory motion are introduced.

The first approach uses an iterative method where each wind component, in- and cross-track, are measured at different points during the oscillation. These distinct points are selected because they exhibit minimum sensitivity to the wind component that is not being measured at that point (thus minimizing the error due to the inaccuracies on the other wind component estimate). These measurements are finally iteratively refined.

The second approach measures both wind components by determining the oscillation's frequency and the spacecraft's attitude during the oscillation's zerocrossings. This approach is conceptually simpler, as it does not require the estimation of the spacecraft's angular acceleration

\subsection{Iterative Approach}

For simplicity, it is assumed that the restoring torque is linear with $\theta_{\mathrm{sf}}^{\mathrm{eq}}=0$ (see Eq. 5), and that the aerodynamic torque $\tau_{\text {aero }}$ is the only torque applied to the spacecraft (no other perturbations considered). In such a simplified case, the governing equation of motion can be expressed as follows:

$J \ddot{\theta}=-q k \theta_{\mathrm{sf}}$

Let the wind $\boldsymbol{w}$ be represented by an in-track component $w_{i}$ and a cross-track component $w_{c}$. The in-track component is aligned with the inertial velocity $\boldsymbol{v}_{\mathrm{i}}$ and the cross-track component is perpendicular to it.

$\|\boldsymbol{w}\|^{2}=w_{i}^{2}+w_{c}^{2}$

The goal of the method is to recover these two components of the wind $\boldsymbol{w}$. To simplify this case even further, without loss of generality, it is assumed that the inertial velocity $\boldsymbol{v}_{i}$ is aligned with the inertial axes so when the spacecraft is aligned with the inertial velocity $\theta_{\mathrm{i}}=0 \Longleftrightarrow \theta=0$.

Another assumption that is made, without loss of generality, is that there is no atmospheric co-rotation. It can be considered that the atmospheric co-rotation is included within the wind measurement, once the wind is estimated the co-rotation can be subtracted to obtain the true wind measurement. Under these assumptions, the dynamic pressure $q$ and the angles $\theta_{\text {sf }}$ or $\theta_{\text {flow }}$ can be written as follows:

$q=\frac{1}{2} \rho\left(\left(v_{i}+w_{i}\right)^{2}+w_{c}^{2}\right)$

$\tan \left(\theta-\theta_{\text {sf }}\right)=\tan \left(\theta_{\text {flow }}\right)=\frac{w_{c}}{v_{i}+w_{i}}$

The magnitudes that are assumed to be known (or assumed to be measurable) are: spacecraft inertia $J$, aerodynamic stiffness $k$, atmospheric density $\rho$, inertial velocity $v_{i}$, spacecraft attitude $\theta$, angular velocity $\dot{\theta}$, and 
angular acceleration $\ddot{\theta}$. At a single point in time, the dynamics of the system can be reduced to a single equation (combining Eq. 8, 10a, and 10b and thus the intrack and cross-track can not be, in principle, measured simultaneously.

In order to compute both wind components the oscillating behavior of the system is exploited. The natural frequency $\omega_{0}$ of this system can be easily computed as

$\omega_{0}=\sqrt{\frac{q k}{J}}$

Although the final objective is to measure both the intrack and the cross-track wind components, it may be initially convenient to explore how the cross-track wind can be computed if the in-track wind component is ignored - set to zero - or known (using a previous estimate). The $(\hat{\cdot})$ notation is used to differentiate the estimated magnitudes from the known or ground truth variables (e.g., $\hat{w}_{i}$ denotes the estimate of the in-track wind).

As the inertial velocity is generally much greater than the in-track wind $v_{i} \gg w_{i}$, assuming that $\hat{w}_{i}=0$ only introduces a small error in $\hat{q}$ and $\hat{\theta}_{\text {flow }}$. After a more accurate estimation of the in-track wind is obtained the results can be updated to obtain more accurate results (forming the basis of the iterative approach).

With the assumption of a known in-track wind component $w_{i}$, the cross-track wind component $w_{c}$ can be iteratively computed at any time during the spacecraft's oscillation using the following set of equations:

$\hat{q}=\frac{1}{2} \rho\left(\left(v_{i}+\hat{w}_{i}\right)^{2}+\hat{w}_{c}^{2}\right)$

$\hat{\omega}_{0}=\sqrt{\frac{\hat{q} k}{J}}$

$\hat{\theta}_{\text {flow }}=\frac{\ddot{\theta}}{\hat{\omega}_{0}^{2}}+\theta$

$\hat{w}_{c}=\left(v_{i}+\hat{w}_{i}\right) \tan \left(\hat{\theta}_{\text {flow }}\right)$

To initialize the iterative algorithm the cross-track wind component can be set to $\hat{w}_{c}=0$, or to any other previously known estimate. Then, by applying Eq. (12a. 12d), the value of $\hat{w}_{c}$ is iteratively updated until it converges. During these iterations, $\hat{w}_{i}$ remains constant at its predefined value $\left(e . g ., \hat{w}_{i}=0\right)$.

This method only converges to the true cross-track wind if the in-track wind estimate $\hat{w}_{i}$ is accurate $\hat{w}_{i}=$ $w_{i}$. As the system oscillates, there is a point where the error introduced by an inaccurate in-track wind estimate $\hat{w}_{i}$ is minimal. The sensitivity of the estimated cross-track wind with respect to the in-track wind $\frac{\partial w_{c}}{\partial w_{i}}$ (shown in Eq. 13) offers a method to select the measurement point of $\hat{w}_{c}$ that minimizes the error induced by an inaccurate $\hat{w}_{i}$.

$\frac{\partial w_{c}}{\partial w_{i}}=\frac{w_{c}}{v_{i}+w_{i}}-\frac{2 \ddot{\theta}}{\omega_{0}^{2}}$

To minimize the error induced by an inaccurate $\hat{w}_{i}$ on $\hat{w}_{c}, \frac{\partial w_{c}}{\partial w_{i}}$ has to be minimized (as this ratio indicates how sensitive the cross-track winds solution is to an error on the in-track wind estimate). During the attitude oscillations caused by the aerostability, where the angular acceleration $\ddot{\theta}$ also oscillates, there are instants when this $\frac{\partial w_{c}}{\partial w_{i}}$ sensitivity is larger than others. Achieving a $\frac{\partial w_{c}}{\partial w_{i}}=0$ completely eliminates the error associated with an inaccurate in-track estimate, as the measurement of the cross-track component is insensitive (i.e., independent) from the in-track wind estimate. This condition is achieved at the following angular acceleration

$\frac{\partial w_{c}}{\partial w_{i}}=0 \Longleftrightarrow \ddot{\theta}=\frac{\omega_{0}^{2} w_{c}}{2\left(v_{i}+w_{i}\right)} \approx \frac{\hat{\omega}_{0}^{2} \hat{w}_{c}}{2\left(v_{i}+\hat{w}_{i}\right)}$

Unfortunately, this point of zero sensitivity depends on the in- and cross-track components and thus it is not accurately known. As $v_{i} \gg w_{i}$ the approximation of Eq. (14) yields acceptable results, even if the in-track component is neglected $\hat{w}_{i}=0$. It is also important to note that the point where $\frac{\partial w_{c}}{\partial w_{i}}=0$ is also dependent on the cross-track component $w_{c}$, which is the magnitude to be measured and thus initially unknown. To solve this problem, the cross-track wind component is continuously estimated during the oscillation. Certain points will satisfy Eq. (14), making the estimates associated with these instants the ones presenting a smaller error. It is also important to note that this minimum sensitivity condition occurs twice every oscillation and thus two good cross-track measurement per oscillation can be taken.

As at this point, good estimates of the cross-track components are available and a similar procedure can be used to estimated the in-track component $\hat{w}_{i}$. By iteratively using Eq. 15a $15 \mathrm{~d}$, and using the previously obtained estimates of the cross-track wind $\hat{w}_{c}$, an estimate of the in-track wind $\hat{w}_{i}$ is obtained.

$\hat{\theta}_{\text {flow }}=\arctan \left(\frac{\hat{w}_{c}}{v_{i}+\hat{w}_{i}}\right)$

$\hat{\omega}_{0}^{2}=\frac{\ddot{\theta}}{\hat{\theta}_{\text {flow }}-\theta}$ 
$\hat{q}=\frac{{\hat{\omega_{0}}}^{2} J}{k}$

$\hat{w}_{i}=\sqrt{\frac{2 \hat{q}}{\rho}-\hat{w}_{c}^{2}}-v_{i}$

Again, as this set of equations assumes a certain crosstrack component (which in general is not completely accurate), an error is introduced when estimating $\hat{w}_{i}$. To minimize it, the measurements can be taken when $\frac{\partial w_{i}}{\partial w_{c}}$ is minimal. It is easy to see from Eq. 13 that $\frac{\partial w_{i}}{\partial w_{c}}$ is minimized when $|\ddot{\theta}|$ is maximized. In this case, the error is not completely eliminated as $\frac{\partial w_{i}}{\partial w_{c}}$ never reaches zero $\left(\frac{\partial w_{i}}{\partial w_{c}}=0 \Longleftrightarrow|\ddot{\theta}|=\infty\right)$. It is also important to note that the maximum acceleration is also achieved twice per oscillation and thus two good measurements per oscillation of the in-track component are available.

With these two iterative processes, the cross- and intrack wind components can be estimated. As the measurement point of the in- and cross-track components is not coincident, the estimates need to be interpolated so that they are available when attempting to obtain the other wind component estimate (i.e., cross-track wind interpolation is required to obtain the cross-track estimate used to estimate the in-track wind).

This whole procedure can be done offline. The data can be first collected by the spacecraft and be later processed. The iterative process in Eq. 12a. 12d and Eq. (15a, $15 \mathrm{~d}$ ) is finally repeated multiple times (also iteratively) subsequently refining the wind estimates and updating their interpolated values.

\subsection{Frequency Approach}

Measuring the frequency of the oscillation provides an alternative approach to measure the wind. If it is assumed that the wind components are averaged over an oscillation (denoted by $\bar{w}_{c}$ and $\bar{w}_{i}$ ), and substituting the dynamic pressure (Eq. 10a and $\theta_{\text {flow }}$ into the equation of motion, the following equation can be obtained:

$J \ddot{\theta}=-\frac{1}{2} \rho\left[\left(v_{i}+\bar{w}_{i}\right)^{2}+\bar{w}_{c}\right] k\left(\theta-\arctan \left(\frac{\bar{w}_{c}}{v_{i}+\bar{w}_{i}}\right)\right)$

Similarly, the natural frequency can be written as follows:

$\omega_{0}=\sqrt{\frac{\rho}{2 J}\left[\left(v_{i}+\bar{w}_{i}\right)^{2}+\bar{w}_{c}^{2}\right]}$
Eq. 16 and (17) can be solved to obtain the averaged wind values over the oscillation's period. The following expressions provide the in-track wind and cross-track wind components for a given oscillation's natural frequency:

$\bar{w}_{i}=\sqrt{\left(\frac{2 J \omega_{0}^{2}}{\rho k}-\bar{w}_{c}^{2}\right)}-v_{i}$

$\bar{w}_{c}=\sqrt{\frac{2 J \omega_{0}^{2}}{\rho k}-\left(v_{i}-\bar{w}_{i}\right)^{2}}$

Substituting the solution for $\bar{w}_{i}$ into the equation of motion gives the following expression:

$\ddot{\theta}=-\omega_{0}^{2}\left(\theta-\arctan \left(\sqrt{\frac{\bar{w}_{c}^{2}}{\frac{2 J \omega_{0}^{2}}{\rho k}-\bar{w}_{c}^{2}}}\right)\right)$

Finally, solving for $\bar{w}_{c}$ gives the following expression, which provides the cross-track wind estimated given the current angle $\theta$, angular acceleration $\ddot{\theta}$, and oscillation frequency $\omega_{0}$ :

$\bar{w}_{c}=\sqrt{\frac{2 J \omega_{0}^{2}}{\rho k} \sin ^{2}\left(\frac{\ddot{\theta}}{\omega_{0}^{2}}+\theta\right)}$

Following the same procedure for $\bar{w}_{i}$ yields the following equation to estimate the averaged in-track wind:

$\bar{w}_{i}=\sqrt{\frac{2 J \omega_{0}^{2}}{\rho k} \cos ^{2}\left(\frac{\ddot{\theta}}{\omega_{0}^{2}}+\theta\right)}-v_{i}$

With these equations, a scheme where measurements are taken four times per oscillation can be imagined. Two measurements are taken when the angular rate is $\operatorname{maximum} \dot{\theta}_{\max }\left(\right.$ where $\theta_{\dot{\theta} \max }=\theta_{\text {flow }}$ and $\left.\ddot{\theta}_{\dot{\theta} \max }=0\right)$. In that case Eq. 21] and (22) can be written as follows:

$\bar{w}_{c}=\sin \left(\theta_{\dot{\theta} \max }\right) \frac{2 \pi}{\tau} \sqrt{\frac{2 J}{\rho k}}$

$\bar{w}_{i}=\cos \left(\theta_{\dot{\theta} \max }\right) \frac{2 \pi}{\tau} \sqrt{\frac{2 J}{\rho k}}-v_{i}$

where $\tau$ denotes the oscillation's period $\tau=2 \pi / \omega_{0}$.

Another two measurements are taken when the angular acceleration $\ddot{\theta}_{\max }$ is maximum (where the angular amplitude with respect to the flow $\theta_{\text {sfmax }}$ is maximum). In this case, the equations can be written as follows:

$\hat{\bar{w}}_{c}=\sin \left(\frac{\ddot{\theta}_{\max } \tau^{2}}{4 \pi^{2}}+\theta_{\max }\right) \frac{2 \pi}{\tau} \sqrt{\frac{2 I}{\rho k}}$ 
$\hat{\bar{w}}_{i}=\cos \left(\frac{\ddot{\theta}_{\max } \tau^{2}}{4 \pi^{2}}+\theta_{\max }\right) \frac{2 \pi}{\tau} \sqrt{\frac{2 I}{\rho k}}-V_{i}$

Note how the oscillation period is required to obtain the averaged estimates. The period can be easily obtained, as four times the time between the maximum angular acceleration and maximum angular rate.

This other approach is not iterative and thus does not require previous knowledge of the in-track or crosstrack wind. Also if the measurements are done when the maximum angular rate is achieved (Eq. 23a and $23 \mathrm{~b}$ there is no need to estimate the angular acceleration, thus significantly simplifying the whole measurement process (an advantage over the iterative approach presented in Section 3.2). Taking measurements during maximum angular rate becomes the default option for the frequency approach and thus two measurements per cycle are obtained.

Unfortunately the assumption that the winds remain constant throughout the oscillation's period is quite limiting. The accuracy of the method can be significantly degraded if the winds variability is not below the natural frequency of the spacecraft. Another source of error may be derived from the damping induced by the spacecraft structural flexibility. This damping has an impact on the oscillation frequency, biasing the wind estimates.

\subsection{Spatial Resolution}

If the wind is roughly constant and the spacecraft exhibits the aerodynamic aerostable response shown in Eq. (5), then the spacecraft oscillates around its equilibrium point. Two in-track and two cross-track wind measurements are available per spacecraft oscillation (sampling frequency is $2 \omega_{0}$ ). Using the Nyquist criterion it is easy to see that this method can recover wind frequencies that are lower than the oscillation frequency of the spacecraft (Eq. 11).

This approach matches the results obtained if the transmissibility of the system is analyzed. As the spacecraft ideally behaves as an undamped oscillator the only excitations (i.e., wind) that have an effect on the spacecraft attitude are those with a natural frequency around or below the spacecraft's natural frequency. The transmissibility gain $G$ with respect to the ratio of the excitation frequency $\omega_{w}$ with the natural frequency $\omega_{0}$ is shown in Fig. 2, This behavior helps to filter out wind variability that is above the measurement frequency threshold (reducing errors). However, the transmissibility gain $G$ peaks around the natural frequency, making clear that the wind frequency shall be below the

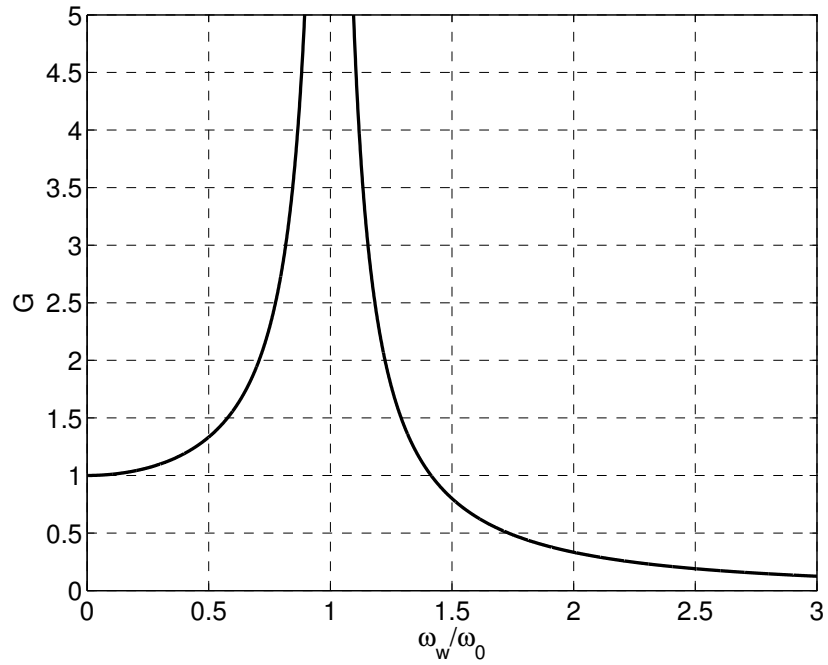

Fig. 2: Transmissibility gain for an undamped oscillator.

spacecraft's natural frequency in order to bound the spacecarft's oscillation. The expected wind variability frequency can be coarsely estimated with current atmospheric models [38, 39, 16].

A perturbation affecting the transmissibility of the system is the one induced by the spacecraft's structural flexibility. A damping effect can also be produced by viscous dampers or hysteresis rods. These damping perturbations slightly shift the angular frequency of the damped system. Given the adverse effects of the damping torques it is recommended to avoid them, by eliminating the presence of damping elements and increasing the rigidity of the spacecraft.

As the spacecraft is traveling across the atmosphere at a significant velocity, the temporal sampling frequency can be related to the measurements spatial resolution $d$. Equation 25 shows how the spatial resolution can be computed (or estimated if the effect of wind and atmospheric co-rotation on the natural frequency is ignored).

$d=\frac{2 \pi\left\|\boldsymbol{v}_{i}\right\|}{\omega_{0}} \approx 2 \pi \sqrt{\frac{2 J}{\rho k}}$

The inertia $J$ and, to a high degree, the aerodynamic stiffness $k$, are only a function of the spacecraft's mass and shape. As expected, low inertia spacecraft with high aerodynamic stiffness (exhibiting higher natural frequencies) provide a better spatial resolution.

The atmospheric density also has a significant effect on the spatial resolution. As the density exponentially increases as the altitude is reduced, the spatial resolution exponentially improves when lowering the spacecraft's operational altitude. 


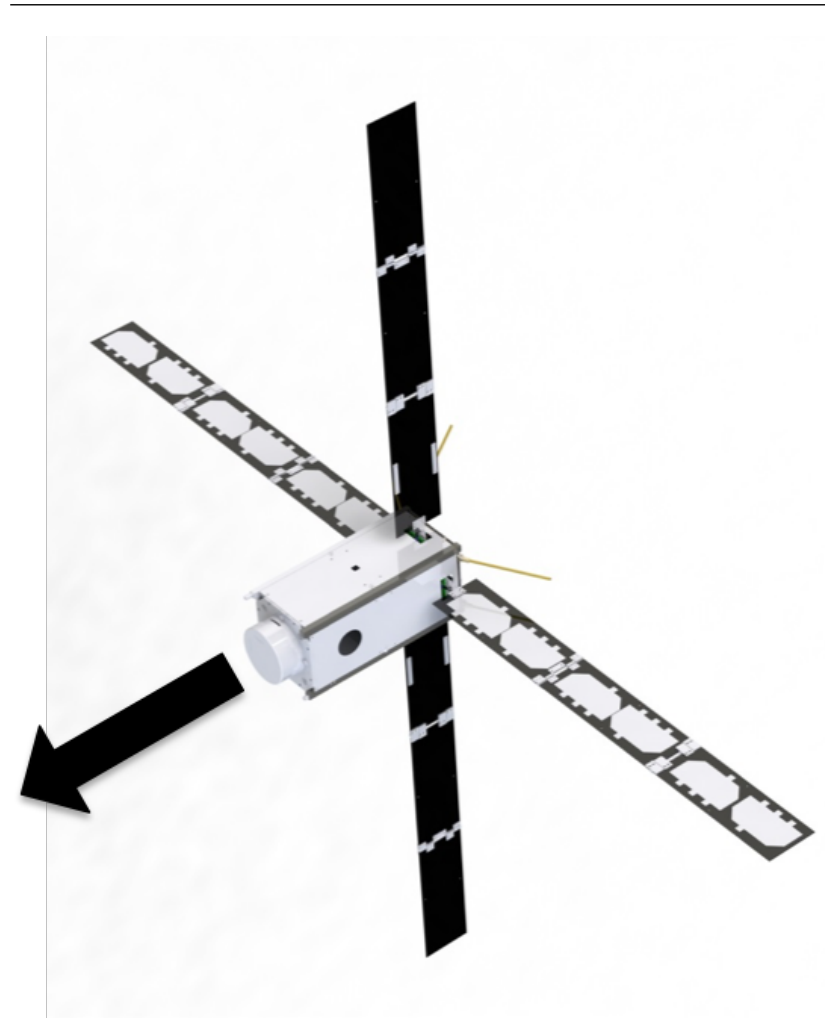

Fig. 3: External configuration of the proposed 2U CubeSat.

Table 1: Proposed CubeSat properties.

\begin{tabular}{cc}
\hline Property & Value \\
\hline Inertia in yaw & $J=0.0318 \mathrm{~kg} \mathrm{~m}^{2}$ \\
Aerodynamic stiffness & $k=0.17 \mathrm{~N} \mathrm{~m} / \mathrm{rad}$ \\
\hline
\end{tabular}

\section{One-Dimensional Case Study}

To illustrate the proposed method, a one-dimensional example is provided. In this example, a proposed $2 \mathrm{U}$ CubeSat 40, partially designed with the objective to recover atmospheric winds (see Fig. 3), is used. The expected properties of this spacecraft are provided in Table 1.

The CubeSat's long appendages, located towards the trailing side of the spacecraft, provide a large aerodynamic stiffness, making it aerostable and allowing it to remain oscillating when left uncontrolled. In this case study, it is assumed that the spacecraft operates in a Keplerian circular orbit and the atmospheric density is estimated using the NRLMSISE-00 model [41] with moderate solar activity [42].

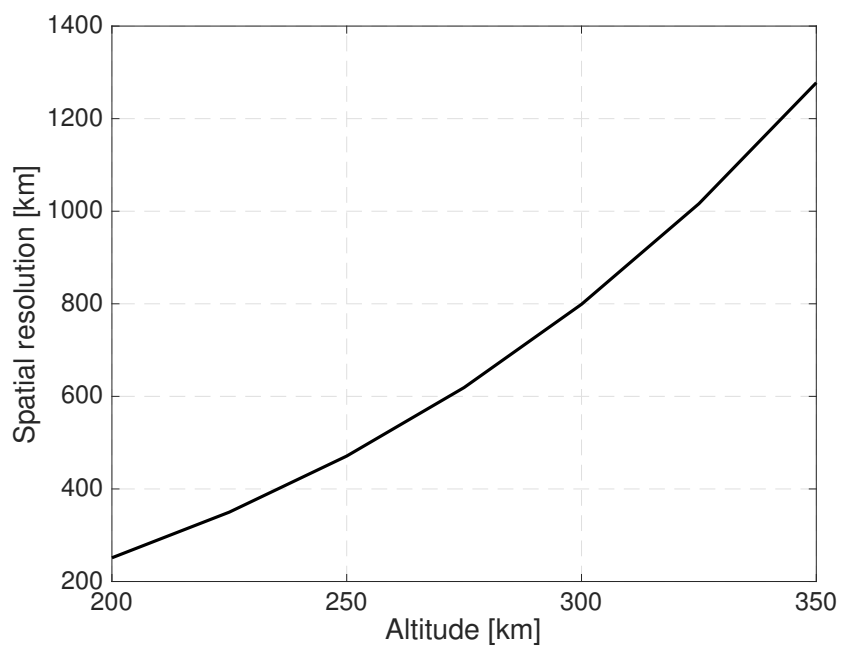

Fig. 4: Spatial resolution of the wind measurements of the proposed CubeSat.

Table 2: Estimation parameters.

\begin{tabular}{cc}
\hline Parameter & Value \\
\hline Operational altitude & $250 \mathrm{~km}$ \\
Initial attitude oscillation amplitude & $10^{\circ}$ \\
In-track wind magnitude & $200 \mathrm{~m} / \mathrm{s}$ \\
Cross-track wind magnitude & $200 \mathrm{~m} / \mathrm{s}$ \\
Attitude Meas. Frequency & $5 \mathrm{~Hz}$ \\
\hline
\end{tabular}

\subsection{Spatial Resolution}

For this specific spacecraft design, the spatial resolution, with respect to the operating altitude, is shown in Fig. 4 (assuming a mean atmospheric density).

\subsection{Ideal Performance}

By assuming a perfect knowledge of the spacecraft parameters and atmospheric density, and in a "noise-free" scenario, the ideal performance of the two methods can be extracted. The parameters used for this ideal wind estimation are shown in Table 2 .

Figure 5 and 6 show the estimation errors for the iterative and frequency approach in this ideal "noisefree" case. These results are obtained for different wind frequencies $\omega_{w}$ (relative to the spacecraft's natural frequency $\left.\omega_{w} / \omega_{0}\right)$. A relative wind frequency of 0 indicates that the wind is constant. When a relative frequency is introduced, the wind component magnitude oscillates (as a sine wave).

From these figures it is clearly noticed that the crosstrack wind can be estimated with smaller errors than the in-track wind. This is an expected result, as when estimating the in-track wind, the full in-track relative velocity is estimated, with the in-track wind just being 


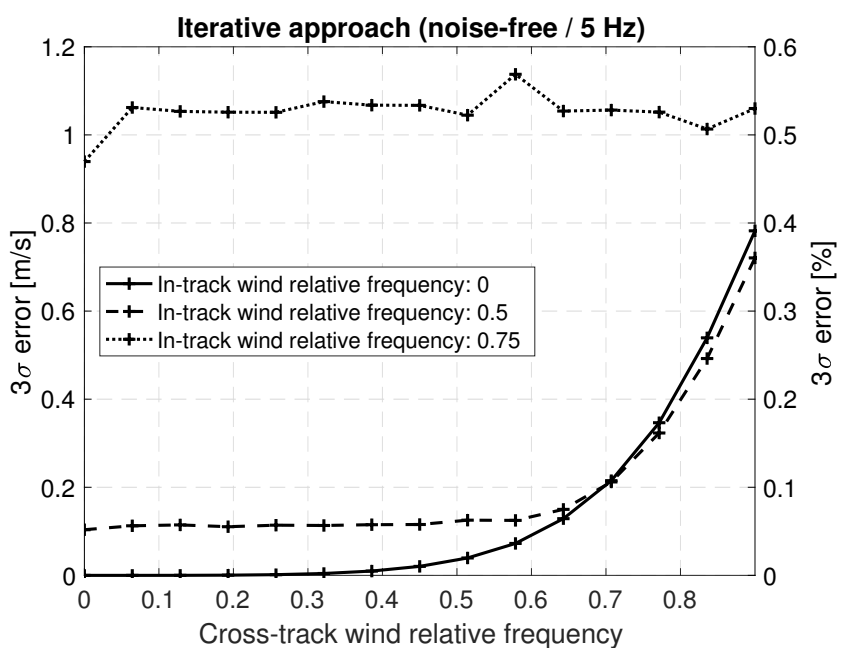

(a) Cross-track wind error $(3 \sigma)$

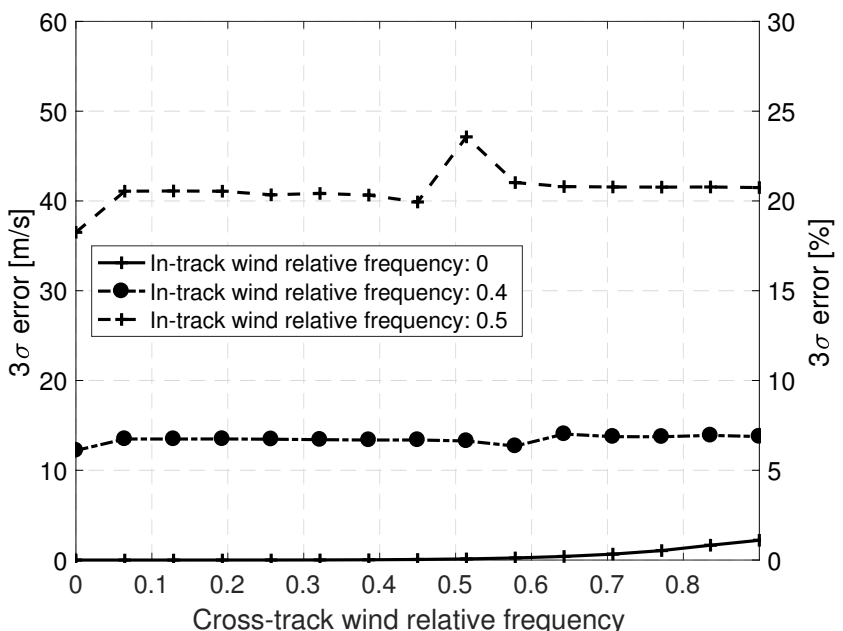

(b) In-track wind error $(3 \sigma)$

Fig. 5: Iterative approach ideal estimation error.

a small part of it $v_{i} \gg w_{i}$. It is also clear that the iterative approach produces more accurate estimates than the frequency approach.

From Fig. 5 and 6 it can also be concluded that, in general, the estimation uncertainties grow larger with higher wind relative frequencies (exception in Fig. 6b). This is also expected, as both methods need to determine the instant in time when these measurements are taken. The advantageous properties of these particular instants are exploited. If the wind magnitude is rapidly changing, these properties are sensitive to the errors in timing. As the attitude measurement frequency is fixed, the "time resolution" is constant, and the resulting timing errors translate to larger wind estimation errors.

This analysis allows us to conclude that higher attitude measurement frequencies, increasing the measurements "time resolution", are desirable. This effect is illustrated in Fig. 7 which shows the cross-track wind es-

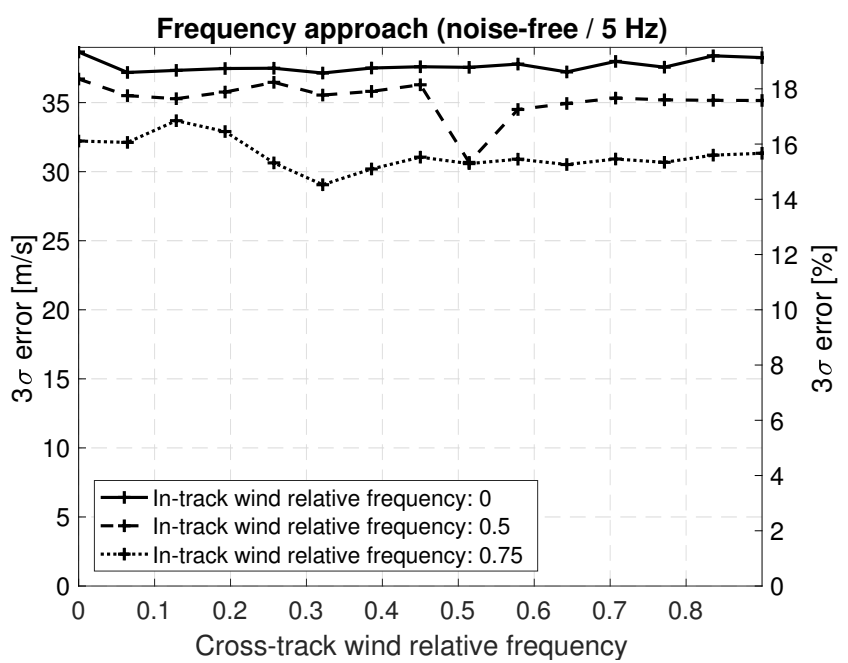

(a) Cross-track wind error $(3 \sigma)$

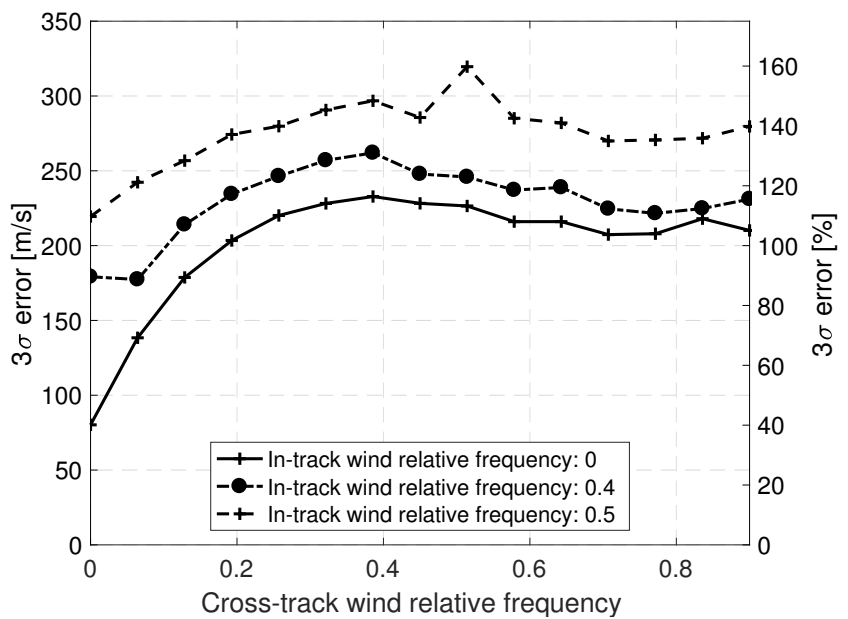

(b) In-track wind error $(3 \sigma)$

Fig. 6: Frequency approach ideal estimation error.

timation uncertainties using the iterative approach but with attitude measurements at $1 \mathrm{~Hz}$. As expected the errors grow when the attitude measurement frequency is decreased.

\subsection{Performance with Uncertainties}

When adding the uncertainties associated with the spacecraft properties or the other measurements (i.e., density, angular velocity, and angular acceleration) the performance of the wind estimation methods decreases.

If the uncertainties provided in Table 3 are added, Fig. 8 and 9, are obtained. These figures show the estimation errors for the iterative and frequency approach in this "noisy" case.

The uncertainty in the natural frequency (see Eq. 11) aggregates the uncertainties on atmospheric density $\rho$, 


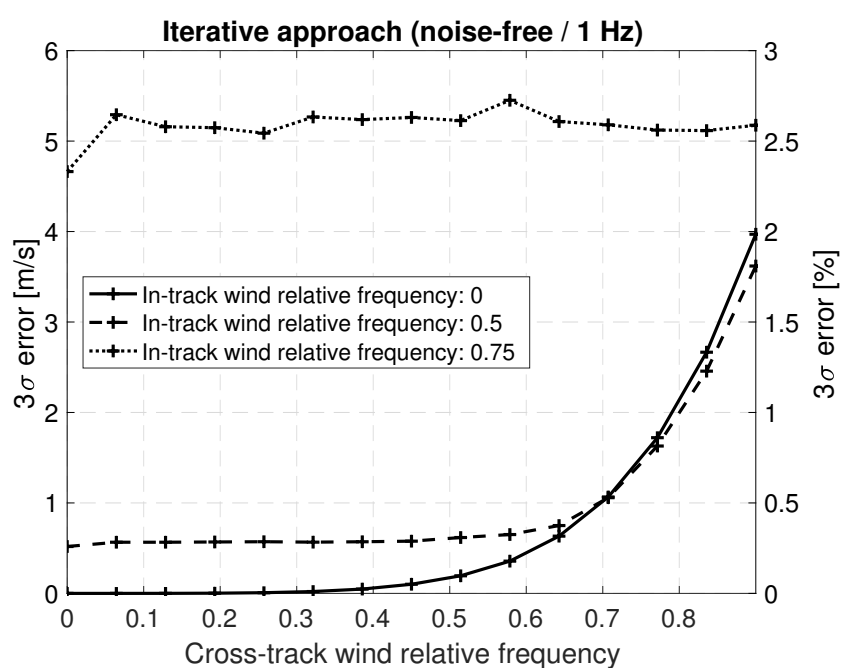

Fig. 7: In-track wind error $(3 \sigma)$ for the Iterative approach ideal case with attitude measurements at $1 \mathrm{~Hz}$.

Table 3: Spacecraft uncertainty parameters.

\begin{tabular}{cc}
\hline Parameter & Uncertainty $(3 \sigma)$ \\
\hline Natural frequency & $20 \%$ \\
Attitude measurement & $10^{\prime \prime}$ \\
Angular acceleration & $100^{\prime \prime} / \mathrm{s}^{2}$ \\
\hline
\end{tabular}

aerodynamic stiffness $k$, and spacecraft inertia $J$. The attitude measurement errors and measurement frequency are based on the ST400 CubeSat star-tracker [4].

These results show that the uncertainties in the intrack wind estimates are probably to large too be useful when there is a moderate amount of in-track relative frequency. However, the cross-track wind can be estimated with remarkable accuracy. Again, the iterative approach produces more accurate estimates than the frequency approach.

\subsection{Comparison to the Accelerometer-Based Approach}

The iterative approach, can obtain cross-track wind estimates with uncertainties well below $10 \mathrm{~m} / \mathrm{s}$. This uncertainty level is very similar to the state-of-the-art of the accelerometer-based approach (see Section 2).

As the proposed method exploits the attitude motion it relies on angular measurement sensors instead of accelerometers. Accurate star-trackers (with errors $<$ $1^{\prime \prime}$ ) and angular-rate gyroscopes (with drifts $<0.003^{\circ} / \mathrm{hr}$ ) are readily available [4]. Also, this proposed method favors spacecraft with a high aerodynamic stiffness over inertia, making nanosatellites a viable option, potentially lowering the mission's cost.

Despite these differences, these two methods are not mutually exclusive and can be used in tandem. In fact

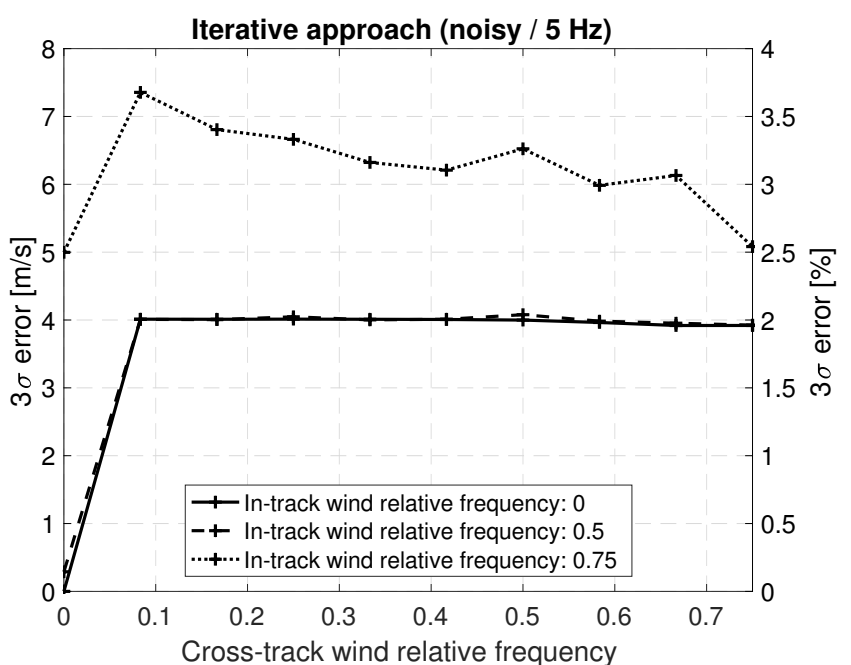

(a) Cross-track wind error $(3 \sigma)$

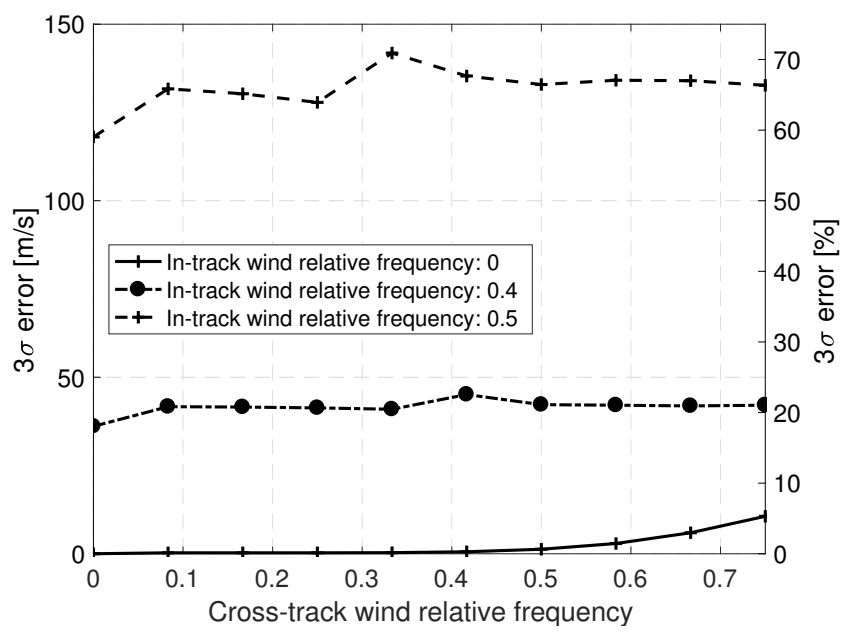

(b) In-track wind error $(3 \sigma)$

Fig. 8: Iterative approach estimation errors parameter uncertainty.

spacecraft using the accelerometer-based measurement method are already equipped with an attitude determination subsystem. The method presented here can be used to re-analyze the data obtained from past missions or inform new designs in order exploit the use of these two methods simultaneously.

\section{Multi-Dimensional Case}

Up to this point, a one-dimensional case has been examined. This allows to extract only one of the two cross-track wind components. The vertical winds are generally smaller [17 than the horizontal winds and thus a one-dimensional measurement approach could be used to obtain the meridional and zonal winds, leaving out the vertical wind component.The method presented 


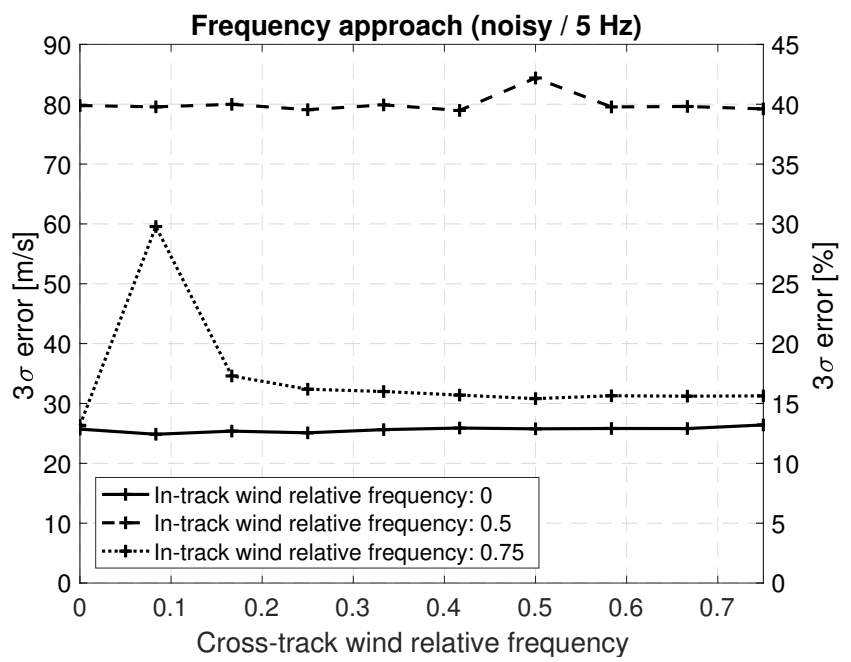

(a) Cross-track wind error $(3 \sigma)$

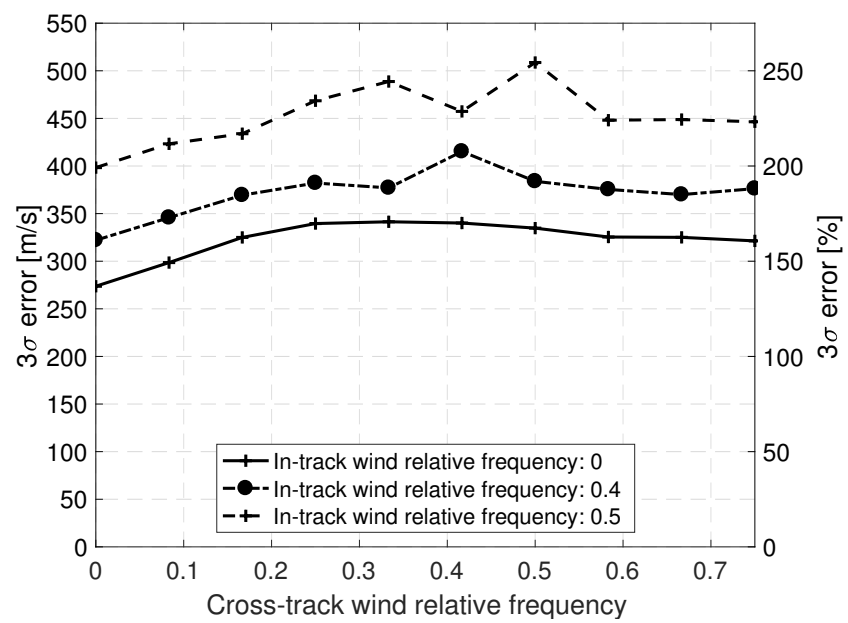

(b) In-track wind error $(3 \sigma)$

Fig. 9: Frequency approach estimation errors with parameter uncertainty.

here can be extended for a multidimensional case and be used to recover the vertical wind.

In a full three-dimensional case, the full Euler's equations need to be used.

$$
I \ddot{\theta}+\dot{\theta} \times I \dot{\theta}=\tau
$$

In addition, the expression of the aerodynamic torque is more complex $\boldsymbol{\tau}_{\text {aero }}=f\left(\boldsymbol{w}, \rho, \boldsymbol{v}_{i}, \boldsymbol{\theta}\right)$ and thus an iterative scheme to find $\boldsymbol{w}$ may be used. As in the onedimensional case, there will be locations where the measurement error will be minimized, but their location will be less obvious. Figure 10 shows a conceptual representation of the overall procedure.

\section{Conclusion}

By observing the attitude evolution of an aerostable spacecraft, the proposed method can estimate the crossand in-track winds. To do so, estimates of the spacecraft's aerodynamic properties and of the atmospheric density are required. The cross-track wind estimates are significantly more accurate than the in-track wind ones. From the two developed approaches, the iterative approach, given its higher accuracy, is the preferred one. The accuracy of the proposed method appears to be better, or at least comparable to, the accuracy of the accelerometer-based approach. By exploiting the attitude response of an aerostable spacecraft to determine winds, the proposed method relies on commercially available attitude sensors (i.e., star-trackers and angular-rate gyroscopes). In contrast, high-precision accelerometers, as the ones required in the accelerometerbased approach are rare, custom made, and expensive.

\section{References}

1. Häusler, K., Lühr, H., Rentz, S., and Köhler, W., "A statistical analysis of longitudinal dependences of upper thermospheric zonal winds at dip equator latitudes derived from CHAMP," Journal of Atmospheric and SolarTerrestrial Physics, Vol. 69, No. 12, 8 2007, pp. 1419 1430. doi $10.1016 /$ j.jastp.2007.04.004

2. Larsen, M. F. and Fesen, C. G., "Accuracy issues of the existing thermospheric wind models: can we rely on them in seeking solutions to wind-driven problems?" $A n$ nales Geophysicae, Vol. 27, No. 6, 2009, pp. 2277-2284. doi 10.5194/angeo-27-2277-2009

3. King-Hele, D., "The upper atmosphere as sensed by satellite orbits," Planetary and Space Science, Vol. 40, No. 23, 1992, pp. 223 - 233. doi $10.1016 / 0032-0633(92) 90060-$ 2 .

4. Merson, R. H., King-Hele, D. G., and Plimmer, R. N. A. "Changes in the Inclination of Satellite Orbits to the Equator," Nature, Vol. 183, No. 4656, 01 1959, pp. 239240.

5. King-Hele, D. and Walker, D. M., "Upper-atmosphere zonal winds from satellite orbit analysis: An update," Planetary and Space Science, Vol. 36, No. 11, 1988, pp. 1085 - 1093. doi 10.1016/0032-0633(88)90062-1.

6. Larsen, M. F., "Winds and shears in the mesosphere and lower thermosphere: Results from four decades of chemical release wind measurements," Journal of Geophysical Research: Space Physics, Vol. 107, No. A8, 2002, pp. SIA 28-1-SIA 28-14. doi 10.1029/2001JA000218.

7. Liu, H., Lühr, H., Watanabe, S., Köhler, W., Henize, V., and Visser, P., "Zonal winds in the equatorial upper thermosphere: Decomposing the solar flux, geomagnetic activity, and seasonal dependencies," Journal of Geophysical Research: Space Physics, Vol. 111, No. A7, 2006, pp. n/a-n/a. doi 10.1029/2005JA011415

8. Sutton, E. K., Nerem, R. S., and Forbes, J. M., "Density and Winds in the Thermosphere Deduced from Accelerometer Data," Journal of Spacecraft and Rockets, Vol. 44, No. 6, 2007, pp. 1210-1219. doi $10.2514 / 1.28641$ 


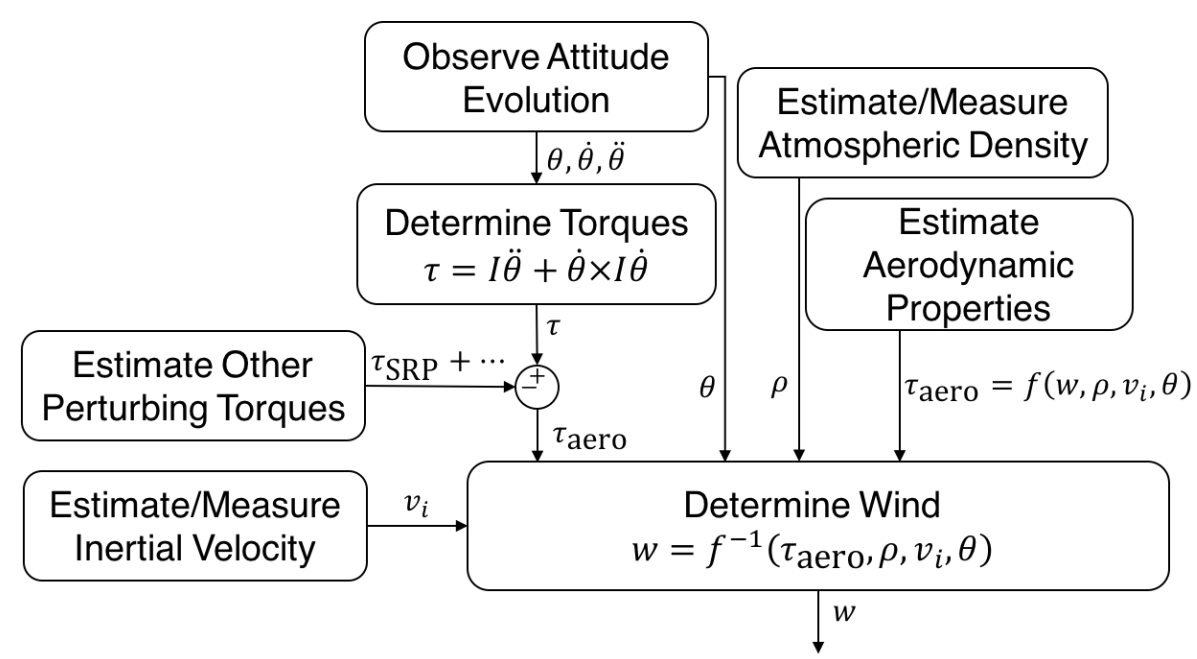

Fig. 10: Conceptual representation of the proposed approach.

9. Doornbos, E., Den Ijssel, J. V., Luehr, H., Foerster, M., and Koppenwallner, G., "Neutral Density and Crosswind Determination from Arbitrarily Oriented Multiaxis Accelerometers on Satellites," Journal of Spacecraft and Rockets, Vol. 47, No. 4, 2015/02/27 2010, pp. 580-589. doi $10.2514 / 1.48114$.

10. Crowley, G. and Tolson, R., "Mars Thermospheric Winds from MGS and Odyssey Accelerometers," AIAA/AAS Astrodynamics Specialist Conference and Exhibit, American Institute of Aeronautics and Astronautics, 2015/03/21 2006. doi doi:10.2514/6.2006-6392

11. Moe, K. and Moe, M., Method for Deriving Densities and In-Track Winds During Storms, American Institute of Aeronautics and Astronautics, 2015/03/21 2006. doi doi:10.2514/6.2006-6396

12. Lühr, H., Rentz, S., Ritter, P., Liu, H., and Häusler, K., "Average thermospheric wind patterns over the polar regions, as observed by CHAMP," Annales Geophysicae, Vol. 25, No. 5, 2007, pp. 1093-1101. doi 10.5194/angeo25-1093-2007

13. Ritter, P., Lühr, H., and Doornbos, E., "Substormrelated thermospheric density and wind disturbances derived from CHAMP observations," Annales Geophysicae, Vol. 28, No. 6, 2010, pp. 1207-1220. doi 10.5194/angeo28-1207-2010

14. Lieberman, R. S., Akmaev, R. A., Fuller-Rowell, T. J., and Doornbos, E., "Thermospheric zonal mean winds and tides revealed by CHAMP," Geophysical Research Letters, Vol. 40, No. 10, 2013, pp. 2439-2443. doi $10.1002 /$ grl.50481.

15. Sivla, W. and McCreadie, H., "Mid-latitude thermospheric zonal winds during the equinoxes," Advances in Space Research, Vol. 54, No. 3, 2014, pp. 499 508. doi 10.1016/j.asr.2014.03.014, Recent Advances in Equatorial, Low- and Mid-Latitude Mesosphere, Thermosphere-Ionosphere System Studies.

16. Drob, D. P., Emmert, J. T., Meriwether, J. W., Makela, J. J., Doornbos, E., Conde, M., Hernandez, G., Noto, J., Zawdie, K. A., McDonald, S. E., Huba, J. D., and Klenzing, J. H., "An update to the Horizontal Wind Model (HWM): The quiet time thermosphere," Earth and Space Science, Vol. 2, No. 7, 2015, pp. 301-319. doi $10.1002 / 2014 \mathrm{EA000089}$
17. Doornbos, E., S. Bruinsma, S., Fritsche, G., Koppenwallner, P., Visser, J., van den IJssel, and de Teixeira de Encarnacao, J., "GOCE+ theme 3:Air density and wind retrieval using GOCE data final report," Tech. Rep. Tech. Rep. 4000102847/NL/EL, TU Delft, Netherlands, 2014.

18. Visser, P., Doornbos, E., van den IJssel, J., and Teixeira da Encarnação, J., "Thermospheric density and wind retrieval from Swarm observations," Earth, Planets and Space, Vol. 65, No. 11, 2013, pp. 1319-1331. doi $10.5047 /$ eps.2013.08.003

19. Spencer, N. and Carignan, G., "In situ measurements of thermospheric composition, temperature and winds by mass spectrometry," Advances in Space Research, Vol. 8, No. 5, 1988, pp. 107 - 117. doi $10.1016 / 0273-$ 1177(88)90040-3.

20. Shepherd, G. G., Spectral imaging of the atmosphere, Vol. 82, Academic press, 2002.

21. Tepley, C., Burnside, R., Jr., J. M., Hays, P., and Cogger, L., "Spatial mapping of the thermospheric neutral wind field," Planetary and Space Science, Vol. 32, No. 4, 1984, pp. 493 - 501. doi 10.1016/0032-0633(84)90128-4

22. Makela, J. J., Fisher, D. J., Meriwether, J. W., Buriti, R. A., and Medeiros, A. F., "Near-continual groundbased nighttime observations of thermospheric neutral winds and temperatures over equatorial Brazil from 2009 to 2012," Journal of Atmospheric and SolarTerrestrial Physics, Vol. 103, No. 0, 2013, pp. 94 102. doi http://dx.doi.org/10.1016/j.jastp.2012.11.019 Recent Advances in Equatorial, Low- and Mid-latitude Aeronomy.

23. Shepherd, G. G., Thuillier, G., Cho, Y.-M., Duboin, M.L., Evans, W. F. J., Gault, W. A., Hersom, C., Kendall, D. J. W., Lathuillère, C., Lowe, R. P., McDade, I. C., Rochon, Y. J., Shepherd, M. G., Solheim, B. H., Wang, D.Y., and Ward, W. E., "The Wind Imaging Interferometer (WINDII) on the Upper Atmosphere Research Satellite: A 20 year perspective," Reviews of Geophysics, Vol. 50, No. 2, 2012, pp. n/a-n/a. doi 10.1029/2012RG000390.

24. Fejer, B. G., Emmert, J. T., and Sipler, D. P., "Climatology and storm time dependence of nighttime thermospheric neutral winds over Millstone Hill," Journal of Geophysical Research: Space Physics, Vol. 107, No. A5, 2002, pp. SIA 3-1-SIA 3-9. doi 10.1029/2001JA000300. 
25. Wu, Q., McEwen, D., Guo, W., Niciejewski, R., Roble, R., and Won, Y.-I., "Long-term thermospheric neutral wind observations over the northern polar cap," Journal of Atmospheric and Solar-Terrestrial Physics, Vol. 70, No. 16, 2008, pp. 2014 - 2030. doi:http://dx.doi.org/10.1016/j.jastp.2008.09.004

26. Gerrard, A. J. and Meriwether, J. W., "Initial daytime and nighttime SOFDI observations of thermospheric winds from Fabry-Perot Doppler shift measurements of the 630-nm OI line-shape profile," Annales Geophysicae, Vol. 29, No. 9, 2011, pp. 1529-1536. doi 10.5194/angeo29-1529-2011

27. Meriwether, J., "Studies of thermospheric dynamics with a Fabry-Perot interferometer network: A review," Journal of Atmospheric and Solar-Terrestrial Physics, Vol. 68, No. 13, 2006, pp. 1576 - 1589. doi $10.1016 /$ j.jastp.2005.11.014, Passive Optics Aeronomy Passive Optics Workshop.

28. Killeen, T. L., Hays, P. B., Spencer, N. W., and Wharton, L. E., "Neutral winds in the polar thermosphere as measured from Dynamics Explorer," Geophysical Research Letters, Vol. 9, No. 9, 1982, pp. 957-960. doi:10.1029/GL009i009p00957

29. Hays, P. B., Killeen, T. L., and Kennedy, B. C., "The Fabry-Perot Interferometer on Dynamics Explorer," Space Science Instrumentation, Vol. 5, Dec. 1981, pp. 395-416.

30. Spencer, N. W., Wharton, L. E., Niemann, H. B., Hedin, A. E., Carrignan, G. R., and Maurer, J. C., "The Dynamics Explorer Wind and Temperature Spectrometer," Space Science Instrumentation, Vol. 5, Dec. 1981, pp. $417-428$.

31. Hays, P. B., Abreu, V. J., Dobbs, M. E., Gell, D. A., Grassl, H. J., and Skinner, W. R., "The highresolution doppler imager on the Upper Atmosphere Research Satellite," Journal of Geophysical Research: Atmospheres, Vol. 98, No. D6, 1993, pp. 10713-10723. doi $10.1029 / 93 J D 00409$.

32. Burrage, M. D., Arvin, N., Skinner, W. R., and Hays, P. B., "Observations of the O2 atmospheric band nightglow by the high resolution Doppler imager," Journal of Geophysical Research: Space Physics, Vol. 99, No. A8, 1994, pp. 15017-15023. doi 10.1029/94JA00791

33. Killeen, T. L., Wu, Q., Solomon, S. C., Ortland, D. A., Skinner, W. R., Niciejewski, R. J., and Gell, D. A., "TIMED Doppler Interferometer: Overview and recent results," Journal of Geophysical Research: Space Physics, Vol. 111, No. A10, 2006, pp. n/a-n/a. doi: $10.1029 / 2005 J A 011484$

34. Salah, J. E. and Holt, J. M., "Midlatitude thermospheric winds from incoherent scatter radar and theory," Radio Science, Vol. 9, No. 2, 1974, pp. 301-313. doi $10.1029 / \mathrm{RS} 009 \mathrm{i} 002 \mathrm{p} 00301$.

35. Lathuillère, C., Lilensten, J., Gault, W., and Thuillier, G., "Meridional wind in the auroral thermosphere: Results from EISCAT and WINDII-O(1D) coordinated measurements," Journal of Geophysical Research: Space Physics, Vol. 102, No. A3, 1997, pp. 44874492. doi $10.1029 / 96$ JA03429

36. Witasse, O., Lilensten, J., Lathuillere, C., and Pibaret, B., "Meridional thermospheric neutral wind at high latitude over a full solar cycle," Annales Geophysicae, Vol. 16, No. 10, 1998, pp. 1400-1409. doi 10.1007/s00585998-1400-3.

37. Prieto, D. M., Graziano, B. P., and Roberts, P. C., "Spacecraft drag modelling," Progress in
Aerospace Sciences, Vol. 64, 2014, pp. 56 - 65 . doi $10.1016 /$ j.paerosci.2013.09.001.

38. Hedin, A. E., Fleming, E. L., Manson, A. H., Schmidlin, F. J., Avery, S. K., Clark, R. R., Franke, S. J., Fraser, G. J., Tsuda, T., Vial, F., and Vincent, R. A., "Empirical wind model for the middle and lower atmosphere." Journal of Atmospheric and Terrestrial Physics, Vol. 58, No. 13, 1996, pp. 1421-1447. doi 10.1016/0021-9169(95)00122-0

39. Drob, D. P., Emmert, J. T., Crowley, G., Picone, J. M., Shepherd, G. G., Skinner, W., Hays, P., Niciejewski, R. J., Larsen, M., She, C. Y., Meriwether, J. W., Hernandez, G., Jarvis, M. J., Sipler, D. P., Tepley, C. A., O'Brien, M. S., Bowman, J. R., Wu, Q., Murayama, Y., Kawamura, S., Reid, I. M., and and, R. A. V., "An empirical model of the Earth's horizontal wind fields: HWM07," Journal of Geophysical Research: Space Physics, Vol. 113, No. A12304, 2008. doi 10.1029/2008JA013668

40. Virgili, J. and Roberts, P. C., "DDsat, a QB50 CubeSat mission to study rarefied-gas drag modelling," Acta Astronautica, Vol. 89, No. 0, 2013, pp. 130 - 138 doi $10.1016 /$ j.actaastro.2013.04.006

41. Picone, J. M., Hedin, A. E., Drob, D. P., and Aikin, A. C., "NRLMSISE-00 empirical model of the atmosphere: Statistical comparisons and scientific issues," Journal of Geophysical Research: Space Physics, Vol. 107, No. A12, 2002. doi 10.1029/2002JA009430.

42. ISO 14222, "ISO 14222 Space environment (natural and artificial). Earth upper atmosphere," Tech. Rep. ISO 14222:2013, ISO, September 2013.

43. Hyperion Technologies B.V., "ST400 Star Tracker Flyer," http://hyperiontechnologies.nl/wp-content/ uploads/2015/08/HTBST-ST400-V1.01_Flyer.pdf March 2007.

44. Wertz, J. R., Everett, D. F., and Puschell, J. J., Space mission engineering: the new SMAD, Microcosm Press, 2011. 